Research Article

\title{
Large Amplitude Free Vibrations of Mindlin Square Plates - A Novel Formulation
}

\author{
K.Meera Saheb ${ }^{\dot{A}} *$ and G.Venkateswara Rao ${ }^{\dot{B}}$ \\ ${ }^{\grave{A}}$ Department of Mechanical Engineering, University college of engineering(A),JNTU Kakinada-533 003, Andhra Pradesh, India \\ ${ }^{\mathrm{B}}$ Department of Mechanical Engineering, Vardhaman College of engineering, Shamshabad, Hyderabad, 501 218, Andhra Pradesh, India.
}

Accepted 10 January 2014, Available online 01 February 2014, Special Issue-2, (February 2014)

\begin{abstract}
A novel formulation to study the large amplitude free vibration behavior of uniform moderately thick square plates with axially immovable edges is presented. The formulation is very simple as the large amplitude free vibration behavior is accurately evaluated knowing the totally unrelated quantities like the tensions developed in the plate due to large deflections and the corresponding buckling load. The basis for this formulation is the recent work of the authors on predicting the free vibration behavior of initially loaded plates. This novel formulation involves three basic steps, through which the large amplitude free vibration behavior of square plates can be very easily and accurately evaluated by following the assumed space mode (accurate single term admissible functions depending on the boundary conditions) approach used by many researches. Numerical results, with emphasis on the fundamental frequency, for the uniform moderately thick square plates, are presented in the digital form in this paper along with the standard solutions of other researchers, to conclusively demonstrate the efficacy of the startling simplicity of the present novel formulation.
\end{abstract}

Keywords: Free vibration etc.

\section{Introduction}

Plates, whether thin or moderately thick, are the most widely used structural members in many aerospace structural elements which are highly optimized. Evaluation of the large amplitude free vibrations of such plates is essential for studying the dynamic behavior of the structural members under the influence of severe dynamic environment. The basic requirement is to find the variation of the frequency of free vibration of the relatively complex moderately thick square plates, for a given mode, with respect to a given maximum amplitude of vibration. Based on this information further investigations on the dynamic behavior of these structural members follow. This paper presents a novel formulation to study the large amplitude free vibrations of moderately thick square plates, where the effects of shear deformation and rotary inertia have to be considered, with all edges simply supported.

The classic work on the topic of large amplitude free vibrations of hinged-hinged beams is initially due to Woinowsky-Krieger who considered the nonlinearity involved in the strain-displacement relation (von-Karman type) taking into account the constant axial tension developed in the beam because of the large deflections with the axially immovable end conditions. This tension term when included in the dynamic equation of equilibrium, makes it nonlinear (hardening type) and the solution is obtained in terms of the elliptic integrals, with an assumed space mode for the hinged-hinged beam.

It is the endeavor of the authors to develop startlingly simple and at the same time accurate and reliable solutions, to some complex problems of practical interest in structural mechanics, and successfully developed such formulations for predicting the fundamental frequency parameter of initially loaded structural members. The author's have been successfully studied large amplitude free vibration behavior of uniform shear flexible beam and thin plates using simple novel formulation. With the same spirit and following the earlier work, the authors have successfully developed a novel formulation for studying the large amplitude free vibrations of uniform moderately thick square plates with immovable edges, where the nonlinearity exists in the strain-displacement relation of von-Karman type. In the present paper a single term space mode approach is used and the effect of the nonlinearity on the fundamental frequency, is studied, following the work of many researchers on this topic. This formulation is called novel because it is based on the knowledge of two totally unrelated quantities like axial tensile loads developed in the moderately thick square plates due to large deflections and the corresponding buckling loads due to the biaxial compressive loads at the edges. The basic steps involved in the present novel formulation are:

i) Presenting briefly, for the sake of completeness, the derivation of an already developed simple design formula 
to obtain the frequency parameters of initially loaded plates,

ii) Applying the design formula given in step (i) to obtain the ratios of the nonlinear to linear radian frequencies in terms of the maximum amplitude of vibration of the square plates by using the tensions developed due to the large deflections as the initial load, where the nonlinear radian frequency is obtained by using the SHM assumption and

iii) Finally, to obtain the ratios of the nonlinear to linear radian frequencies by eliminating the SHM assumption using the harmonic balance method[ (HBM), that provides a correction factor which corrects the error induced because of the SHM assumption.

The present formulation is mainly based on the physical concepts of the problem and corresponding logical deductions without much complex mathematical treatment. Following these three basic steps, the numerical results of a relatively complex problem of the large amplitude free vibrations of isotropic moderately thick square plates with immovable edges are presented in the digital form. Solution for the case of all edges simply supported are demonstrated and given along with other researchers using complex mathematical formulations are used to assess the accuracy, reliability and simplicity of the present novel formulation. The major three steps, mentioned earlier, used in the proposed formulation are briefly presented in the following sections.

\section{Initially Loaded Free Vibrations of Plates}

The linear free vibration behavior of square plates due to externally applied concentrated axial load can be accurately evaluated following the analysis methods like the Rayleigh-Ritz method, weighted residual methods, corresponding finite element formulations etc. The final governing equation of equilibrium is obtained in the matrix form, assuming SHM, as

$[\mathrm{K}]\{\delta\}-\lambda_{\mathrm{i}}[\mathrm{G}]\{\delta\}-\lambda_{\mathrm{f}}[\mathrm{M}]\{\delta\}=0$

It can be seen from Eq. (1) that for a positive (compressive) initial load, the plate is less stiff and the corresponding natural frequencies decrease and when the initial load is negative (tensile) the structural system gets stiffened and the corresponding natural frequencies increase.

From Eq. (1), the degenerate case of the buckling equation, by neglecting the mass matrix, is given by

$[\mathrm{K}]\left\{\delta_{1}\right\}=\lambda_{\mathrm{b}}[\mathrm{G}]\left\{\delta_{1}\right\}$

Equation (2) can be written as

[G] $\left\{\delta_{1}\right\}=\left(1 / \lambda_{\mathrm{b}}\right)[\mathrm{K}]\left\{\delta_{1}\right\}$

Similarly, the degenerate case for the free vibration problem without the initial load can be obtained from Eq. (1), by neglecting the geometric stiffness matrix, as

$[\mathrm{M}]\left\{\delta_{2}\right\}=\left(1 /^{\lambda} f_{0}\right)[\mathrm{K}]\left\{\delta_{2}\right\}$

Substituting Eqs. (3) and (4) in Eq. (1), we get
$[\mathrm{K}]\{\delta\}-\frac{\lambda_{i}}{\lambda_{b}}[K]\left\{\delta_{1}\right\}-\frac{\lambda_{f}}{\lambda_{f_{o}}}[K]\left\{\delta_{2}\right\}=0$

with a reasonable and valid assumption, particularly for the first buckling and free vibration modes,

$\left\{\delta_{1}\right\} \approx\left\{\delta_{2}\right\} \approx\{\delta\}$

Equation (5) becomes

$[\mathrm{K}]\{\delta\}-\frac{\lambda_{i}}{\lambda_{b}}[K]\{\delta\}-\frac{\lambda_{f}}{\lambda_{f_{o}}}[K]\{\delta\}=0$

or $[\mathrm{K}]\{\delta\}-[K]\{\delta\}\left(\lambda_{i} / \lambda_{b}+\lambda_{f} / \lambda_{f_{o}}\right)=0$

Equation (8) implies that

$\frac{\lambda_{i}}{\lambda_{b}}+\frac{\lambda_{f}}{\lambda_{f_{o}}}=1$

\section{Ratios of Nonlinear to Linear Radian Frequencies with SHM Assumption}

The parameters $\lambda_{\mathrm{f}}$ and $\lambda_{f_{0}}$ in Eq. (9) can be interpreted as $\lambda_{N L_{H}}$, the nonlinear frequency parameter and $\lambda_{\mathrm{L}}\left(\alpha_{m} \rightarrow\right.$ $0)$, the linear frequency parameter and the initial load parameter $\lambda_{i}$ as $\lambda_{\mathrm{T}}$ (tensile) arising due to large deflections as mentioned in the previous section. With these interpretations, Eq. (9) can be written as

$\frac{\lambda_{N L_{H}}}{\lambda_{L}}=\frac{\omega_{N L_{H}}^{2}}{\omega_{L}^{2}}=1+\frac{\lambda_{T}}{\lambda_{b}}$

\section{Correction to Ratios of Nonlinear to Linear Radian} Frequencies Obtained with SHM Assumption

The procedure to evaluate of $\omega_{N L_{H} /} \omega_{L}$ is given in the previous section, with the assumption of SHM, which is not realistic, as the large amplitude free vibration behavior, even though periodic, does not exhibit SHM. However, the linear free vibrations follow the SHM. The following mathematical treatment, applying the HBM[10], gives a correction factor of $3 / 4$, which compensates the error caused because of the assumption of SHM in obtaining the ratios of $\omega_{N_{L}} / \omega_{L}$

\section{Application of Novel Formulation to all Edges Simply Supported Moderately Thick Square Plate}

The procedure to evaluate of non dimensional Tension Parameter is given as follows

The tension developed in the plate in $\mathrm{x}$-direction as

$T_{x}=\frac{E h}{2 a} \int_{0}^{a}\left\{\frac{d w(x)}{d x}\right\}^{2} d x$

$w=\alpha \sin \frac{\pi x}{a} \sin \frac{\pi y}{a}$

Substituting Eq.(12) in Eq.(11) and after simplification

$T_{x}=\frac{E h}{4} \alpha^{2} \frac{\pi^{2}}{a^{2}}$

Substituting $D=\frac{E h^{3}}{12\left(1-v^{2}\right)}$ in the above equation 
Table1: Buckling load parameters $\left(\lambda_{b}\right)$ for all edges simply supported moderately thick square plate with different thickness $\operatorname{ratios}(\mathrm{h} / \mathrm{a})$

\begin{tabular}{|l|l|c|c|c|c|}
\hline $\mathrm{h} / \mathrm{a}$ & 0.001 & 0.05 & 0.1 & 0.15 & 0.2 \\
\hline$\lambda_{b}$ & 2 & 1.9722 & 1.8932 & 1.7748 & 1.6319 \\
\hline
\end{tabular}

Table 2: $\mathrm{T}_{\mathrm{NL}} / \mathrm{T}_{\mathrm{L}}$ values of all edges simply supported square plate for fundamental mode.

\begin{tabular}{|c|c|c|c|c|c|c|c|c|c|c|c|c|}
\hline h/a & \multicolumn{10}{|c|}{$\begin{array}{c}\alpha_{m} \\
h\end{array}$} \\
\hline & \multicolumn{2}{|c|}{0} & \multicolumn{2}{|c|}{0.2} & \multicolumn{2}{|c|}{0.4} & \multicolumn{2}{|c|}{0.6} & \multicolumn{2}{c|}{0.8} & 1.0 \\
\hline & PS & $\begin{array}{c}\text { FEM } \\
{[12]}\end{array}$ & PS & $\begin{array}{c}\text { FEM[ } \\
12]\end{array}$ & PS & FEM[12] & PS & FEM[12] & PS & FEM[12] & PS & FEM[12] \\
\hline 0.001 & 1 & 1 & 0.9801 & 0.9818 & 0.9270 & 0.9327 & 0.8548 & 0.8652 & $\begin{array}{c}0.777 \\
3\end{array}$ & 0.7916 & 0.7029 & 0.7199 \\
\hline 0.05 & 1 & 1 & 0.9799 & 0.9837 & 0.9260 & 0.9290 & 0.8532 & 0.8586 & $\begin{array}{c}0.775 \\
1\end{array}$ & 0.7827 & 0.7005 & 0.7098 \\
\hline 0.1 & 1 & 1 & 09791 & 0.9788 & 0.9233 & 0.9226 & 0.8484 & 0.8475 & $\begin{array}{c}0.768 \\
7\end{array}$ & 0.7681 & 0.6931 & 0.6931 \\
\hline 0.15 & 1 & 1 & 0.9777 & 0.9766 & 0.9188 & 0.9153 & 0.8406 & 0.8350 & $\begin{array}{c}0.758 \\
5\end{array}$ & 0.7520 & 0.6814 & 0.6752 \\
\hline 0.2 & 1 & 1 & 0.9758 & 0.9740 & 0.9126 & 0.9069 & 0.8300 & 0.8212 & $\begin{array}{c}0.744 \\
7\end{array}$ & 0.7347 & 0.6660 & 0.6562 \\
\hline
\end{tabular}

Table3: Buckling load parameters $\left(\lambda_{b}\right)$ for all edges clamped moderately thick square plate with different thickness $\operatorname{ratios}(\mathrm{h} / \mathrm{a})$

\begin{tabular}{|c|c|c|c|c|c|}
\hline $\mathrm{h} / \mathrm{a}$ & 0.001 & 0.05 & 0.1 & 0.15 & 0.2 \\
\hline$\lambda_{b}$ & 5.3333 & 5.3263 & 4.7018 & 4.0541 & 3.4260 \\
\hline
\end{tabular}

Table 4: $T_{N L} / T_{L}$ values of all edges clamped square plate for fundamental mode.

\begin{tabular}{|c|c|c|c|c|c|c|c|c|c|c|c|c|}
\hline \multirow[t]{3}{*}{$\mathrm{h} / \mathrm{a}$} & \multicolumn{12}{|c|}{$\frac{\alpha_{m}}{h}$} \\
\hline & \multicolumn{2}{|r|}{0} & \multicolumn{2}{|c|}{0.2} & \multicolumn{2}{|c|}{0.4} & \multicolumn{2}{|c|}{0.6} & \multicolumn{2}{|c|}{0.8} & \multicolumn{2}{|c|}{1.0} \\
\hline & PS & FEM[12] & PS & FEM[12] & PS & FEM[12] & PS & FEM[12] & PS & FEM[12] & PS & FEM[12] \\
\hline 0.001 & 1 & 1 & 0.9924 & 0.9930 & 0.9706 & 0.9729 & 0.9373 & 0.9421 & 0.8960 & 0.9037 & 0.8501 & 0.8611 \\
\hline 0.05 & 1 & 1 & 0.9923 & 0.9927 & 0.9701 & 0.9718 & 0.9363 & 0.9399 & 0.8943 & 0.9004 & 0.8479 & 0.8567 \\
\hline 0.1 & 1 & 1 & 09919 & 0.9918 & 0.9685 & 0.9684 & 0.9331 & 0.9331 & 0.8894 & 0.8899 & 0.8413 & 0.8427 \\
\hline 0.15 & 1 & 1 & 0.9911 & 0.9902 & 0.9659 & 0.9624 & 0.9278 & 0.9213 & 0.8814 & 0.8720 & 0.8308 & 0.8195 \\
\hline 0.2 & 1 & 1 & 0.9902 & 0.9879 & 0.9623 & 0.9541 & 0.9207 & 0.9050 & 0.8705 & 0.8481 & 0.8167 & 0.7893 \\
\hline
\end{tabular}

$$
\begin{aligned}
& T_{x}=\frac{1}{4} \alpha^{2}\left(\frac{\pi}{a}\right)^{2} \frac{12 D\left(1-v^{2}\right)}{h^{2}} \\
& T_{x}=3\left(\frac{\alpha}{h}\right)^{2}\left(\frac{\pi}{a}\right)^{2} D\left(1-v^{2}\right)
\end{aligned}
$$

The tension developed in the plate does not contain any shear flexible terms.

The non-dimensional tension parameter from the above expression as

$$
\lambda_{T}=\frac{T_{x} a^{2}}{\pi^{2} D}=\left(\frac{\alpha}{h}\right)^{2} 3\left(1-v^{2}\right)
$$

The non dimensional buckling load parameter when square plate subjected to biaxial compression along the edges ( the edges $\mathrm{x}=0, \mathrm{x}=\mathrm{a}$ subjected $N_{x}$ per unit length and the edges $\mathrm{y}=0, \mathrm{y}=\mathrm{a}$ subjected $N_{y}$ per unit length

$$
\lambda_{b}=\frac{N_{x} a^{2}}{\pi^{2} D}=\frac{2}{1+5.6398\left(\frac{h}{a}\right)^{2}}
$$

\section{Numerical Results and Discussion}

As the present formulation to evaluate the large amplitude free vibration behavior of structural elements requires a) the axial tensile loads developed due to the large deflections and b) the buckling load parameter obtained consistent with the tensile loads developed, i.e., edge compressive loads, which are evaluated by suitably 
selecting the single term admissible functions with one undetermined coefficient for the transverse displacement w. Further, for a formulation like the one presented here that is mainly based on the physical concepts and subsequent logical conclusions, it is essential to show how the values of $\omega_{N L_{H}} / \omega_{L}$ obtained initially and $\omega_{N L} / \omega_{L}$ computed using the correction factor match with the FEM[ existing solution, available in the open literature. Buckling loads which are required to evaluate large amplitude free vibration behavior are given in Table1with all edges simply supported and for different thickness ratios and are given in Table1. The present results compare very well with those obtained using the finite element formulation and are given in Table 2 for all edges simply supported square plate. It is worth mentioning here that the present formulation directly gives the ratios of $\omega_{N L_{H}} / \omega_{L}$ and the ratios $\omega_{N L} / \omega_{L}$ are obtained by correcting these values using HBM. Similar buckling loads and time period ratios are given in respectively in table 3 and Table 4 for all edges clamped square plate and found that the present results and FEM results are very closely matching.

\section{Conclusions}

The efficacy of the proposed novel formulation for studying the large amplitude free vibrations of moderately thick square plates has been conclusively demonstrated in this paper. The present formulation is startling simple because it is primarily based on the physical concepts involved in the problem and subsequent logical deductions with very little mathematics, whereas, the earlier studies on this topic are highly mathematically oriented. The authors are of the opinion that the present formulation, which involves three simple basic steps, attracts both the analysts and researchers belonging to the basic engineering research. Most of the researchers on this topic concentrated on the first mode of vibration (fundamental frequency) while studying the large amplitude free vibration phenomenon, assuming simple but accurate single term admissible functions. Hence, the authors are also taken the single term admissible functions with one undetermined coefficient and studied the large amplitude free vibration behavior of moderately thick square plates. The results obtained by present simple formulation very closely matches with the FEM and is given in Table 2 and Table4.

\section{Acknowledgements}

The author is thankful to the authorities of University College of Engineering(A), JNTU-Kakinada for sponsoring the necessary economical support for presenting the paper under TEQIP phase2. Second author gratefully acknowledges the support given by INAE during the course of this work.

\section{References}

Woinowsky-Krieger, S.,(1950) The Effect of an Axial Force on the Vibration of pp.35-36.
G.V.Rao, , and N.R. Naidu, (2001) Prediction of Fundamental Frequencies of Stressed Spring-Hinged Tapered Beams, AIAA J.,39, pp.86-188.

G.V. Rao, (2001), Simple Formula to Predict the Fundamental Frequency of Initially Stressed Square Plates, J. Sound Vib., 246, pp.185-

G.V. Rao, andK.K. Raju, (2002) "Simple Formula for Evaluating the Fundamental Frequency Parameter of Initially Stressed Uniform Beams on Elastic Foundation, Fire Technol., 124, pp.4

G.V.Rao, and R.Neetha, (2003) Prediction of Fundamental Frequency of Initially In plane Loaded Thick Circular Plates, J. Sound Vib., 259, pp.1265-1268.

G.V.Rao, S. Renjit, and G.Rajeev Nair, (2004) Design Formula to Evaluate the Fundamental Frequency of Thin Annular Plates Subjected to a Uniform Compressive Load at the outer Edge, Advances in vibration Engineering, 3, pp.152-160

G.V.Rao, and S.Renjit, (2005) Applicability of the Design Formula for Predicting the Fundamental Frequency of Initially Stressed Moderately Thick Annular Plates, Journal of Structural Engineering, 32, pp.319-325.

G. V. Rao, K.Meera Saheb and G. Ranga Janardhana, (2008) Simple formula to Study the large Amplitude free Vibrations of Beams and Plates, J.Appl.Mech., 75, pp.1-4.

G.V.Rao, K. Meera Saheb, andG. Ranga Janardhana, (2007) Large Amplitude Free Vibrations of Uniform Timoshenko Beams- A Novel Formulation, AIAA J., 45, pp.2810-2812.

L.Azrar, and R.G.White, (1999) A Semi Analytical Approach to the Nonlinear Dynamic Response Problems of S-S and C-C Beams at Large Vibration Amplitudes, Part I: General Theory and Application to the Single Mode Approach to Free and Forced Vibration Analysis, Journal of Sound and Vibration, 224, pp.183-207.

K. Meera Saheb, G.V. Rao, andG. Rangajanardhana, (2009) Buckling and Free Vibration of Moderately Thick Square Plates Using Coupled Displacement Field Method, Journal of structural Engineering,36,PP.155-159.

K.K. Raju, G.V. Rao, andI.S. Raju, (1978) Effect of Geometric Nonlinearity on The Free Flexural Vibrations of Moderately Thick Rectangular Plates, computers and structures, 9, pp.441-444

\section{Nomenclature}

a $\quad=$ square plate dimention

$\alpha_{m}=$ maximum amplitude at the centre of the square plate

[G] = system geometric stiffness matrix

$\mathrm{I}=$ area moment of inertia

$[\mathrm{K}]=$ elastic stiffness matrix

$\mathrm{m}=$ mass of the beam per unit length

$[\mathrm{M}]=$ system mass matrix

$T_{N L}=$ non linear time period

$T_{L} \quad=\quad$ linear time period

$T_{X} \quad=\quad$ tension developed in $\mathrm{x}$ direction due to large deflections

$\{\delta\}=$ eigenvector (vibration mode shape with initial load)

$\left\{\delta_{1}\right\}=$ eigenvector (buckling mode shape)

$\left\{\delta_{2}\right\}=$ eigenvector (vibration mode shape without initial

load)

$\lambda_{\mathrm{b}}=\operatorname{buckling}$ load parameter $\left(\frac{N_{x c r} a^{2}}{D}\right)$

$\lambda_{\mathrm{f}}=$ frequency parameter with initial load $\left(\frac{\rho \omega^{2} a^{4}}{D}\right)$

$\lambda_{f_{0}}=$ frequency parameter without initial load $\left(\frac{\rho \omega_{0}^{2} a^{4}}{D}\right)$ 


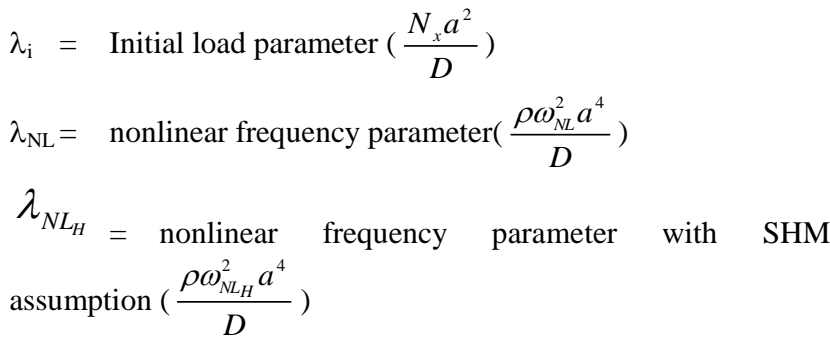

$\lambda_{\mathrm{i}}=\operatorname{Initial}$ load parameter $\left(\frac{N_{x} a^{2}}{D}\right)$

$\lambda_{\mathrm{NL}}=$ nonlinear frequency parameter $\left(\frac{\rho \omega_{N L}^{2} a^{4}}{D}\right)$

$\lambda_{N L_{H}}=$ nonlinear frequency parameter with SHM

assumption $\left(\frac{\rho \omega_{N L_{H}}^{2} a^{4}}{D}\right)$

$\lambda_{\mathrm{T}}=$ axial tensile load parameter $\left(\frac{T_{x} a^{2}}{\pi^{2} D}\right)$

$v=$ Poission's ratio (taken as 0.3 )

$\omega_{f}=$ linear frequency parameter with initial load

$\omega_{N L}=$ nonlinear radian frequency without SHM assumption

$\omega_{0}=$ frequency parameter without initial load

$\omega_{L} \quad=$ linear radian frequency

$\omega_{N L_{H}}=$ nonlinear radian frequency with SHM assumption 